\title{
Imaging Agonist-Induced D2/D3 Receptor Desensitization and Internalization In Vivo with PET/fMRI
}

\author{
Christin Y Sander ${ }^{*, 1,2}$, Jacob M Hooker ${ }^{1,2}$, Ciprian Catana ${ }^{1,2}$, Bruce R Rosen ${ }^{1,2,3}$ and Joseph B Mandeville \\ 'A. A. Martinos Center for Biomedical Imaging, Department of Radiology, Massachusetts General Hospital, Charlestown, MA, USA; ' ${ }^{2}$ larvard \\ Medical School, Boston, MA, USA; ${ }^{3}$ Health Sciences and Technology, Harvard-MIT, Cambridge, MA, USA
}

\begin{abstract}
This study investigated the dynamics of dopamine receptor desensitization and internalization, thereby proposing a new technique for noninvasive, in vivo measurements of receptor adaptations. The D2/D3 agonist quinpirole, which induces receptor internalization in vitro, was administered at graded doses in non-human primates while imaging with simultaneous positron emission tomography (PET) and functional magnetic resonance imaging ( $\mathrm{MMRI}$ ). A pronounced temporal divergence between receptor occupancy and fMRI signal was observed: occupancy remained elevated while fMRI responded transiently. Analogous experiments with an antagonist (prochlorperazine) and a lower-affinity agonist (ropinirole) exhibited reduced temporal dissociation between occupancy and function, consistent with a mechanism of desensitization and internalization that depends upon drug efficacy and affinity. We postulated a model that incorporates internalization into a neurovascular-coupling relationship. This model yielded in vivo desensitization/internalization rates (0.2/min for quinpirole) consistent with published in vitro measurements. Overall, these results suggest that simultaneous PET/AMRI enables characterization of dynamic neuroreceptor adaptations in vivo, and may offer a first non-invasive method for assessing receptor desensitization and internalization. Neuropsychopharmacology (2016) 4I, |427-1436; doi:I0.1038/npp.2015.296; published online 2I October 2015
\end{abstract}

\section{INTRODUCTION}

Receptor desensitization and internalization (RDI) are synaptic mechanisms that modulate downstream cellular activity in response to G-protein-coupled receptor (GPCR) activation by an agonist. RDI has been demonstrated in vitro for many GPCR systems, including dopamine (DA) D2 receptors (D2Rs; Guo et al, 2009). Measurements in in vitro systems suggest that the timeframe of internalization is relatively fast (within minutes of agonist exposure), and receptors may stay internalized for a much longer time period (several hours or days; Skinbjerg et al, 2010). These data suggest that synaptic adaptation mechanisms affect pharmacodynamics in vivo and thus need to be considered for optimizing drug doses and strategies to minimize side effects. Being able to measure RDI and evaluate how its dynamics affect drug action and function in vivo would be important to optimize therapeutic treatment for neurologic and neuropsychiatric diseases. However, methods for assessing RDI non-invasively in living mammalian brain are currently unavailable.

*Correspondence: Dr CY Sander, A. A. Martinos Center for Biomedical Imaging, Department of Radiology, Massachusetts General Hospital, Harvard Medical School, I49 Thirteenth Street, Room 230I, Charlestown, MA 02129, USA, Tel: +617 724 1839, Fax: +617 726 7422, E-mail: csander@mgh.harvard.edu

Received 28 April 2015; revised 14 September 2015; accepted 14 September 2015; accepted article preview online 21 September 2015
In the context of drug-receptor interactions, the classical occupancy theory postulates that receptors can be either in a bound or unbound state at the postsynaptic membrane, and that binding to or unbinding from receptors causes a functional response (Clark, 1937; Maehle et al, 2002). In positron emission tomography (PET) blocking or competition studies, the occupancy model carries the assumption that changes in radiotracer binding potential directly reflect availability of a given synaptic receptor density and, that receptor affinities do not change. This model provides a valid explanation for some experimental data: for certain PET ligands, decreases in binding potential accompany increases in DA concentration, as measured by microdialysis (Breier et al, 1997; Laruelle et al, 1997). A tight relationship between endogenous DA measured from microdialysis and functional magnetic resonance imaging (fMRI) signal changes is also concordant with the classical model (Chen et al, 1997, 1999; Choi et al, 2006), though fMRI signal changes can be affected by different subtypes of receptors (Mandeville et al, 2013). In addition, similar temporal responses from fMRI and PET receptor occupancy due to D2 antagonism (Sander et al, 2013b) support the classical occupancy theory.

Despite the above findings, there are a number of reported experimental observations that cannot be explained by the classical occupancy theory. One consistent observation is that the decrease in binding potential of $\mathrm{D} 2 \mathrm{R}$ antagonist radiotracers due to amphetamine lasts much longer than the time course of the drug, or the efflux of extracellular DA. Moreover, radiotracers such as spiperone and pimozide 
(non-benzamides) show 'paradoxical'-binding properties that oppose those predicted from the classical occupancy theory (Chugani et al, 1988). As proposed previously (Chugani et al, 1988; Ginovart, 2005; Laruelle, 2000), such contradictions to the classical theory are likely due to agonist-induced receptor internalization. The latter can influence ligand-specific-binding rates by altering receptorligand affinity.

Simultaneous functional imaging with PET and fMRI provides a novel platform to study functional receptor dynamics in vivo. Together, the two imaging modalities enable concurrent measurements of receptor occupancy and hemodynamic parameters, which reflect induction of GPCR cellular functions downstream from ligand-receptor complexes. With dynamic data acquisitions and analyses, changes in biochemical and functional parameters can be tracked simultaneously in time. Targeting the DA system pharmacologically with a D2/D3 antagonist leads to PET and fMRI signal changes that are matched in time, which provides evidence that hemodynamics are directly linked to receptor occupancy by neurovascular-coupling mechanisms under these conditions (Sander et al, 2013b). However, in agonist-induced competition studies, we postulated that receptor adaptation mechanisms would alter the functionoccupancy relationship in time.

The purpose of this study was to investigate receptor adaptation mechanisms and in vivo RDI indices noninvasively using simultaneous PET/fMRI. First, a biophysical model of agonist-induced RDI is proposed, together with a signal model that shows how PET and FMRI data are affected. We hypothesized that RDI prolongs the decrease in binding potential from $\left[{ }^{11} \mathrm{C}\right]$ raclopride but shortens the fMRI temporal response and diminishes its magnitude compared with results that would be obtained in the absence of RDI. Second, imaging experiments with simultaneous $\left[{ }^{11} \mathrm{C}\right]$ raclopride-PET and fMRI in non-human primates (NHP) were performed using injections of graded doses of two D2 agonists. The temporal and dose responses allow a novel assessment of agonist-induced receptor adaptation mechanisms in vivo with the proposed PET/fMRI methods.

\section{MATERIALS AND METHODS}

\section{Theory}

According to the classical occupancy theory, receptor occupancy by agonist causes a downstream functional response, which can be observed with PET/fMRI. Highaffinity agonists are known to cause receptor internalization (Goggi et al, 2007; Lane et al, 2012), and thus we expect the classical theory to be an insufficient description. Although in vitro studies have detailed mechanisms of receptor internalization, a model for an in vivo functional readout that links non-invasive measurements to RDI has not been established. Here we describe the main model and how it relates to experimental in vivo measurements of RDI. More details with derivations are described in Supplementary Information.

PET and FMRI signal models. The functional hemodynamic response (measured as changes in cerebral blood volume (CBV) in this study) to a D2 agonist can be expressed in terms of changes in receptor occupancies of the agonist (ligand L) and endogenous DA, which is displaced according to the law of mass action. The functional CBV signal can be modeled through a direct coupling relationship with occupancy (Sander et al, 2013b):

$\Delta \mathrm{CBV}(t)=N_{\mathrm{D} 2} B_{\max } \theta_{\mathrm{L}}(t)\left(\varepsilon_{\mathrm{L}}-\theta_{\mathrm{DA}}^{(0)}\right)$.

$N_{\mathrm{D} 2}$ denotes the neurovascular-coupling constant at the $\mathrm{D} 2 \mathrm{R}, B_{\max }$ the total density of receptors, $\theta_{\mathrm{L}}$ the occupancy of $\mathrm{L}$ and $\theta_{\mathrm{DA}}^{(0)}$ the basal DA occupancy. Drug efficacy is described by $\varepsilon_{\mathrm{L}}$, which is 0 for an antagonist and 1 for a full agonist. Equation (1) describes a steady-state model that is not directly dependent on any affinities or rate constants and assumes a linear approximation between changes in occupancies of DA and ligand. A general expression and derivation of equation (1) is provided in Supplementary Information (Supplementary Equations 1 and 2).

Receptor occupancy, as measured by PET, is derived from baseline and drug challenge binding potentials and by taking into account the relative affinity $\alpha$ (ratio of affinity to internal and external receptors) of the radiotracer:

$\theta_{\mathrm{PET}}=\frac{\theta_{\mathrm{L}}+\left(\theta_{\mathrm{DA}}-\theta_{\mathrm{DA}}^{(0)}\right)+(1-\alpha) \theta_{\mathrm{int}}}{\left(1-\theta_{\mathrm{DA}}^{(0)}\right)}$

Equation (2) expresses occupancy as a sum of receptors bound externally by $\mathrm{L}$ and DA, and internally by $\mathrm{L}$. The relative affinity can take the values $\alpha \geq 0$ and thus specify whether internalized receptors are accessible to the radiotracer.

Dynamic occupancy models. Figure 1 illustrates the components of the RDI model and their relationship towards the classical occupancy model. The parameter $\theta_{\text {int }}$ describes the fraction of internalized receptors and $k_{\mathrm{DI}}$ denotes the RDI rate constant. The model reduces to the classical occupancy model for $k_{\mathrm{DI}}=0$. The same model can thus describe how antagonist and agonist occupancies are related to the changes in $\mathrm{CBV}$, under the assumption that no receptor adaptation mechanisms occur. The mathematical model and its assumptions are described in Supplementary Information (Supplementary Equation 3a-d). This dynamic model plus the measurement models for PET and fMRI are a full description of the RDI model employed in this study for simulation and parameter estimation.

\section{Animal Model}

Two animals (male rhesus macaques: M1 (8 years) and M2 (6 years)) underwent PET/MRI in 12 separate studies. For each study, the animal was anesthetized, initially with $10 \mathrm{mg} / \mathrm{kg}$ ketamine and $0.5 \mathrm{mg} / \mathrm{kg}$ xylazene, and maintained with isoflurane ( $1 \%$, mixed with oxygen) after intubation. Physiological parameters (blood pressure, pulse, end-tidal $\mathrm{CO}_{2}$, breathing rate, and oxygen saturation) were continuously monitored throughout the study. All studies and procedures complied with the regulations of the Institutional Animal Care and Use Committee at Massachusetts General Hospital. 


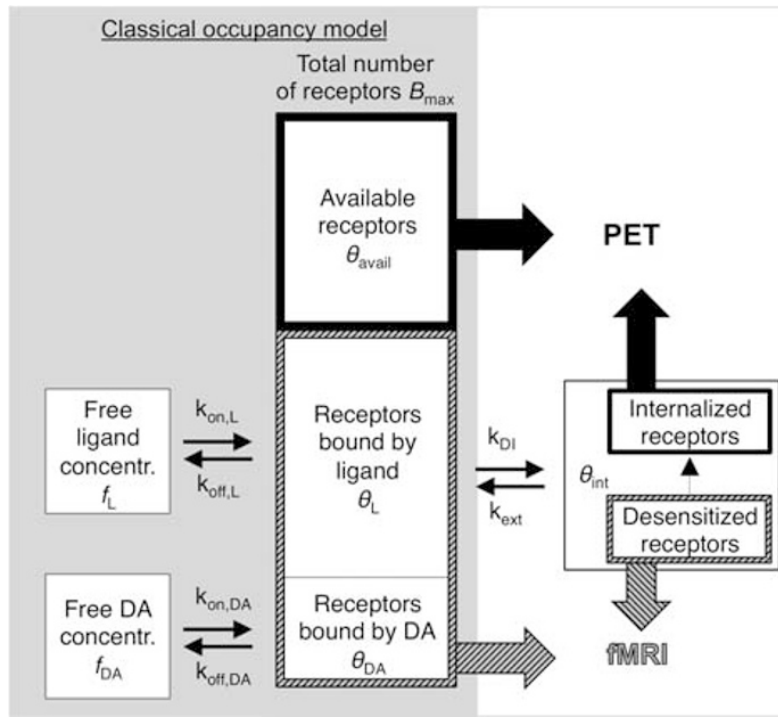

Figure I Schematic illustrating a compartmental model that describes receptor desensitization and internalization at dopaminergic synapses. The total number of receptors $\left(B_{\max }\right)$ is composed of available receptors at the postsynaptic membrane, those bound by an injected agonist, those bound by endogenous dopamine and desensitized/internalized receptors. Occupied receptors are in exchange with free ligand in the synaptic space. Receptors that are occupied by an agonist trigger desensitization and internalization. As externalization mechanisms are known to be very slow, we assume that $k_{\text {ext }}=0$ for the duration of time courses we modeled. The parameters that determine the PET and $\mathrm{PMRI}$ signal changes are highlighted in bold. This shows that PET and fMRI time courses contain complementary information about receptor adaptation mechanisms. concentr., concentration; DA, dopamine; $\mathrm{fMRI}$, functional magnetic resonance imaging; PET, positron emission tomography.

\section{Study Design}

$\left[{ }^{11} \mathrm{C}\right]$ Raclopride was injected using a bolus+infusion protocol. Infusions employed $k_{\mathrm{bol}}$ values (Carson et al, 1993) of 52 and $81 \mathrm{~min}$ on average for animals M1 and M2, respectively. Boluses were administered by hand over a duration of $30 \mathrm{~s}$, after which infusion at a rate of $0.01 \mathrm{ml} / \mathrm{s}$ was started with an automatic pump (Medrad Spectra Solaris). Specific activities at time of injection were $3.7 \pm 2.8 \mathrm{mCi} / \mathrm{nmol}$. At $\sim 30 \mathrm{~min}$, the high-affinity $\mathrm{D} 2 / \mathrm{D} 3$ agonist quinpirole $\left(\mathrm{K}_{\mathrm{D}, \mathrm{D} 2}=576 \mathrm{nM}\right.$; Sokoloff et al, 1990) was injected at one of three different doses $(0.1,0.2$, and $0.3 \mathrm{mg} / \mathrm{kg}$ ), selected to span a range of occupancies from $\sim 30-80 \%$. Quinpirole was chosen as a ligand that reliably induces internalization in vitro. Experiments and repeated administration of pharmacological challenges in all NHPs were separated by at least 2 weeks, so that results were not influenced from prior history. In addition, the order of injections was permuted in animal M1 (highest dose given first) versus animal M2 (highest dose given last) to eliminate potential bias from dose injections. To test whether a single quinpirole injection desensitizes the fMRI response to subsequent injections, the quinpirole injection was repeated $70-120 \mathrm{~min}$ after the first dose in two sessions in animal M2 $(0.1$ and $0.2 \mathrm{mg} / \mathrm{kg})$. The agonist quinpirole was compared with a clinically utilized D2/D3 agonist ropinirole, thought to be of lower affinity $\left(\mathrm{K}_{\mathrm{D}, \mathrm{D} 2}=970 \mathrm{nM}\right.$; Perachon et al, 1999). We hypothesized that a lower-affinity agonist would reduce the rate at which receptors desensitize. In two separate experimental sessions, ropinirole was injected at 0.1 and $0.3 \mathrm{mg} / \mathrm{kg}$ in animal $\mathrm{M} 2$. In addition, the $\mathrm{D} 2 / \mathrm{D} 3$ antagonist prochlorperazine was injected at 0.1 and $0.2 \mathrm{mg} / \mathrm{kg}$ in animal M2 to serve as a direct comparison with the agonists, and to investigate whether the temporal relationship between occupancy and function that was reported for the short-acting antagonist raclopride (Sander et al, 2013b) was maintained for an antagonist with a longer duration of action. Although the employed ligands have affinity for both D2 and D3, the results in caudate and putamen, as determined in this study, are dominated by $\mathrm{D} 2$ as the $\mathrm{D} 2 / \mathrm{D} 3$ ratio in caudate and putamen is 95\% (Cumming, 2011; Narendran et al, 2006).

\section{PET/MR Image Acquisition and Reconstruction}

Simultaneous PET and MR data were acquired on a prototype scanner that consists of a BrainPET insert and a Tim Trio 3T MR scanner (Siemens AG, Healthcare Sector, Erlangen Germany). A custom-built PET compatible eight-channel NHP receive array (Sander et al, 2013a) together with a vendor-supplied local circularly polarized transmit coil was used for MRI. The phased array enabled twofold acceleration with GRAPPA (Griswold et al, 2002) in the anterior-posterior direction. Whole-brain fMRI data were acquired for the duration of PET imaging with multi-slice echo-planar imaging (EPI) that had an isotropic resolution of $1.3 \mathrm{~mm}$ and a temporal resolution of $3 \mathrm{~s}$. Other parameters included $\mathrm{FOV}_{\mathrm{MR}}=110 \times 72.8 \mathrm{~mm}^{2}$, $\mathrm{BW}=1350 \mathrm{~Hz}$ per pixel, flip angle $=60^{\circ}$ and an echo time of $23 \mathrm{~ms}$. To improve fMRI detection power, ferumoxytol (Feraheme, AMAG Pharmaceuticals, Cambridge MA) was injected at $10 \mathrm{mg} / \mathrm{kg}$ before fMRI (Mandeville, 2012).

PET emission data were acquired in list-mode format for 100 min starting with radiotracer injection. Images were reconstructed with a standard 3D Poisson ordered-subset expectation maximization algorithm using prompt and variance-reduced random coincidence events. Normalization, scatter, and attenuation sinograms (including attenuation of the radiofrequency coil) were included in the reconstruction (Catana et al, 2010). The reconstructed volume consisted of $1.25 \times 1.25 \times 1.25-\mathrm{mm}$ voxels in a $256 \times 256 \times 153$ matrix, which were downsampled by a factor of two post reconstruction. Framing intervals were $10 \times 30 \mathrm{~s}$, followed by 1 -min frames.

\section{PET and fMRI Data Analysis}

PET and MR data were registered to the Saleem-Logothetis stereotaxic space (Saleem and Logothetis, 2006) with an affine transformation (12 degrees of freedom, DOF) using a multi-subject MRI template (McLaren et al, 2009), in which standard regions of interest were defined based on anatomy. Alignment of the EPI data used an affine transformation plus local distortion fields. After motion-correcting (AFNI software; Cox, 2012) and spatially smoothing fMRI data with a 2.5-mm Gaussian kernel, statistical analysis was carried out using the general linear model (GLM). The temporal response to the drug injection was modeled with a gamma 


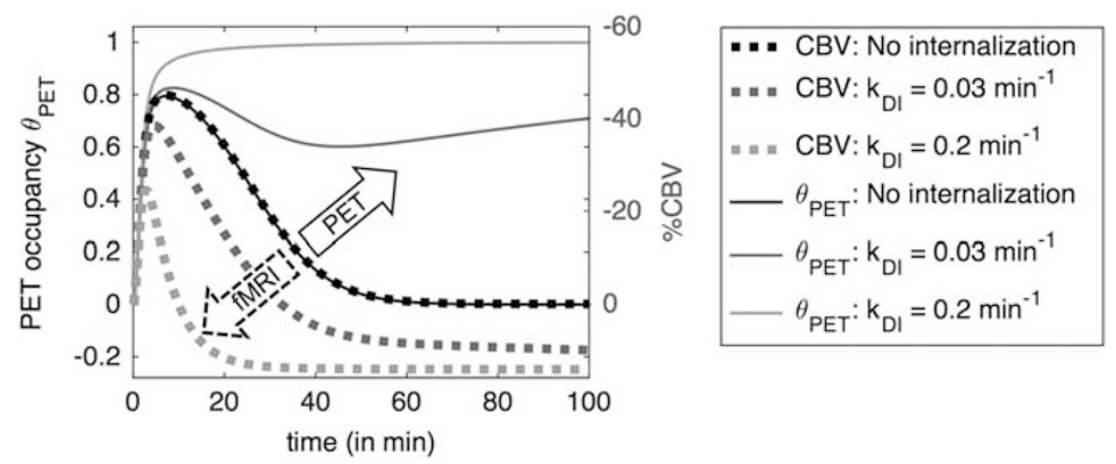

Figure 2 Simulation results from the proposed model of receptor desensitization and internalization that show how PET and fMRI signal time courses are affected for different rates of RDI $\left(\mathrm{k}_{\mathrm{DI}}\right)$ due to a D2/D3 agonist injection at time $t=0$. If no RDI occurs, PET and fMRI signals are matched in time. If RDI occurs with a moderate rate of $0.03 / \mathrm{min}$, PET and $\mathrm{PMRI}$ signals start to diverge. With high RDI rates $(0.2 / \mathrm{min})$, the $\mathrm{fMRI}$ time course is shortened, whereas PET occupancy stays elevated for much longer. CBV, cerebral blood volume; $\mathrm{AMRI}$, functional magnetic resonance imaging; PET, positron emission tomography; $\mathrm{RDI}$, receptor desensitization and internalization.

variate function, in which the time to peak was adjusted to minimize the $\chi^{2} / \mathrm{DOF}$ of the GLM fit to the data. A longlasting signal change that was distributed in several brain regions and not generally specific to the striatum was modeled with a sigmoidal GLM regressor but not included in the temporal drug profile due to its non-specificity. In addition, regressors that correspond to translations in three dimensions and were derived from motion correction were used in the GLM analysis. The resulting signal changes were converted to percent changes in CBV by standard methods (Mandeville et al, 1998).

PET kinetic modeling employed a GLM formulation of the simplified reference tissue model (SRTM) (Lammertsma and Hume, 1996), with the whole cerebellum, excluding the vermis, as the reference ROI and the rate constant $\left(k_{2}\right)$ for the cerebellum derived from the high-binding region putamen, using a two-parameter SRTM model (Ichise et al, 2003). As binding does not stay constant but is dynamically altered as a result of the D2 agonist drug challenge, our kinetic analysis included the time-dependent parameter $k_{2 \mathrm{a}}(\mathrm{t})$ (Alpert et al, 2003; Normandin et al, 2012), which was converted to a 'dynamic-binding potential' (DBP; Sander et al, 2013b).

All PET and fMRI data analysis and generation of parametric images from voxelwise kinetic modeling were generated with open-access software (www.nitrc.org/pro jects/jip). In first-level fixed-effects analyses, all doses exhibited significant responses in putamen and caudate by both PET and fMRI. Statistical values used for maps were computed by regularizing the random effects variance using about 100 effective DOF in the mixed-effects analysis (Worsley et al, 2002).

\section{Estimation of In vivo RDI Constants}

The RDI model was used to generate a family of CBV curves for RDI rates in the range of $0.1-0.03 / \mathrm{min}$. An estimate of the RDI constant for each dataset was then achieved by minimizing the residual norm between the model and data after drug injection.

\section{RESULTS}

\section{Simulations of the Dynamic Occupancy Model and PET/ fMRI Signal}

We investigated how RDI affects the functional (CBV) response, and PET temporal profiles through simulations of the dynamic occupancy model (Equations 1 and 2). Unless specified otherwise, we assumed a full agonist with efficacy 1 , and a radiotracer that does not bind to internalized receptors $(\alpha=0)$. Figure 1 illustrates the entire compartmental model and its relationship with PET and PMRI signals.

Figure 2 shows the simulation results for three RDI rates. Without RDI (black curves, $k_{\mathrm{DI}}=0$ ), $\mathrm{CBV}$ follows the time course of receptor occupancy by the drug. If agonist-induced RDI occurs with RDI rates $>0.01 / \mathrm{min}(0.03$ and $0.2 / \mathrm{min}$ are shown), the PET and CBV temporal responses diverge noticeably on the timescale of a typical $\left[{ }^{11} \mathrm{C}\right]$-PET experiment: the fMRI signal duration is shortened and PET occupancy stays elevated for a longer time when compared with the drug occupancy in the absence of RDI. The temporal divergence increases as RDI rates increase. Without $\mathrm{RDI}$, the CBV time course peaks at $7 \mathrm{~min}$ and returns to baseline at $56 \mathrm{~min}$, consistent with the occupancy of drug. A high RDI rate of $0.2 /$ min (light gray curves) causes the CBV peak to be shifted to $2.8 \mathrm{~min}$ and fall below baseline at $10 \mathrm{~min}$. In addition, the magnitude of the CBV signal decreases as RDI constants become shorter. Peak occupancies of $80 \%$ would be expected to evoke a peak CBV signal of $-45 \%$ without RDI, based upon prior antagonist studies and given the assumption that basal DA occupancy is about $20 \%$ (see Supplementary Information for more details on this assumption). However, simulated peak CBV amplitudes decrease to -39 and $-25 \%$ with RDI rates of 0.03 and $0.2 / \mathrm{min}$, respectively. Higher RDI rates consistently reduce CBV magnitude at all occupancy levels in simulations.

Predictions of the model on how drug efficacy and affinity changes of the radiotracer to internalized receptors affect CBV and PET signals are presented in Supplementary Information (Supplementary Figures 3 and 4). 


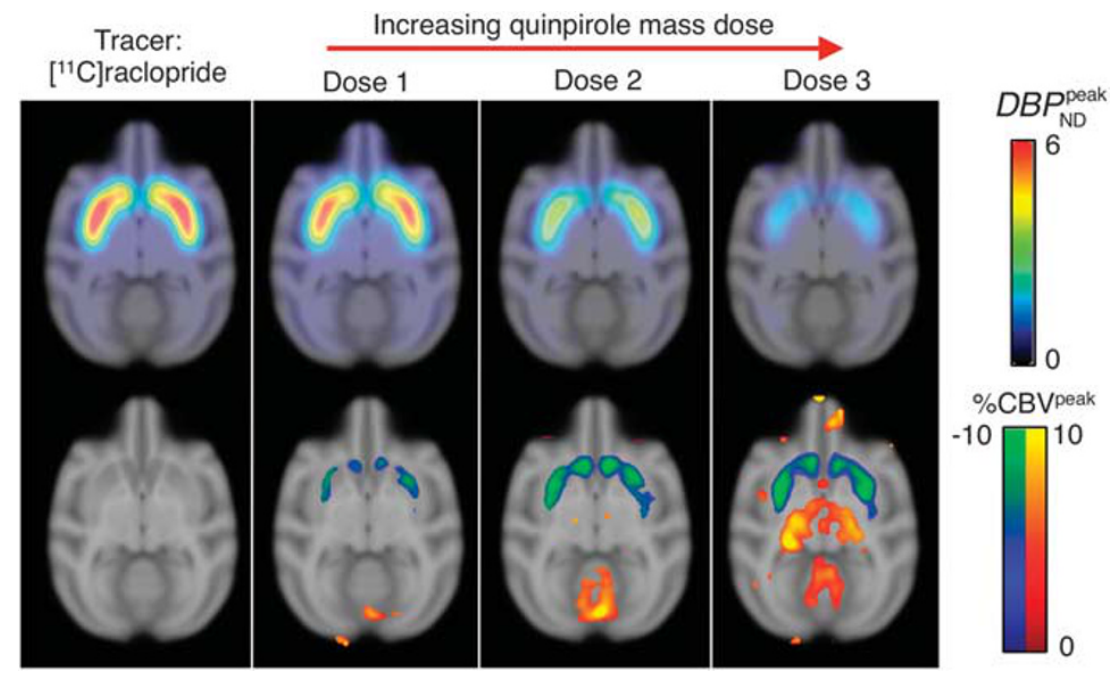

Figure 3 Parametric maps showing the results from three injection doses of the agonist quinpirole. All maps show imaging data from two animals, analyzed with a mixed-effects model. Upper row: dynamic-binding potential maps (DBPReak) show that specific binding of the radiotracer [ $\left.{ }^{\prime \prime} \mathrm{C}\right]$ raclopride in caudate and putamen decreases with increasing quinpirole. Lower row: voxelwise maps showing \%CBVpeak changes, windowed by a $P$-value map with $P<0.03$. As quinpirole dose increases, the negative CBV signal that is specific to caudate and putamen increases in magnitude. CBV, cerebral blood volume; DBP, dynamicbinding potential.
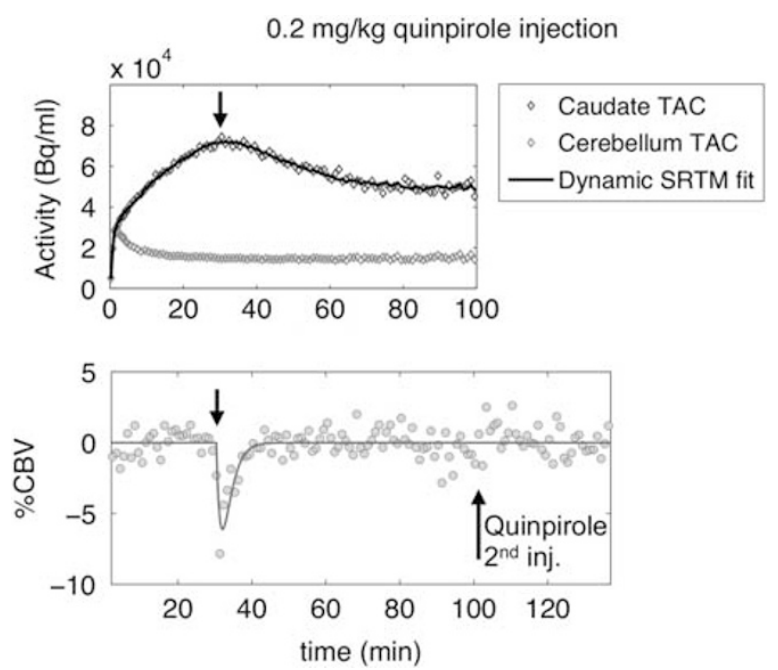

Figure 4 Upper: PET time activity curves for the caudate and cerebellum ROls for the $0.2 \mathrm{mg} / \mathrm{kg}$ quinpirole injection at $35 \mathrm{~min}$ (for animal M2), with kinetic modeling fits from two-parameter SRTM with the cerebellum as the reference. The arrow at $35 \mathrm{~min}$ indicates the time at which the quinpirole challenge was administered. Lower: corresponding CBV time courses show a negative response due to the challenge in the caudate $\mathrm{ROI}$. A second injection of $0.2 \mathrm{mg} / \mathrm{kg}$ quinpirole in the same session did not produce a measurable CBV response in the caudate. CBV, cerebral blood volume; PET, positron emission tomography; SRTM, simplified reference tissue model.

\section{In vivo Experimental Outcomes}

Dose response from occupancy and $C B V$. Parametric maps from PET kinetic modeling results (DBP ${ }_{\mathrm{ND}}^{\text {peak }}$ maps) and fMRI statistical analysis (CBV ${ }^{\text {peak }}$ maps) from three doses of quinpirole injections in two anesthetized animals are shown in Figure 3. Ropinirole injections showed a similar spatial distribution. Specific binding from PET and negative CBV changes both showed a localized response in putamen and caudate. Positive CBV changes were observed for higher doses outside of the striatum, in thalamus and vermis of the cerebellum. The measurements from both modalities were dose dependent: specific binding decreased with increasing dose of quinpirole, whereas CBV signal showed a progressively larger magnitude with increasing dose. Quantitative values from the putamen ROI are listed in Supplementary Table 1 (Supplementary Information) for each quinpirole and ropinirole dose.

Supplementary Figure 1 (Supplementary Information) compares peak $\mathrm{CBV}$ responses $\left(\mathrm{CBV}^{\text {peak }}\right)$ with peak occupancies in putamen and caudate from animal M2. Values from all quinpirole and ropinirole doses for two animals are listed in Supplementary Table 1. Data points were described with a power law fit $\left(a\left(\theta^{\text {peak }}\right)^{b}\right)$ to illustrate that $\mathrm{CBV}^{\text {peak }}$ changes exhibit a monotonically decreasing function versus the occupancy of D2/D3 agonist.

Temporal response from quinpirole doses. Figure 4 shows a representative PET time activity curve (TAC) for caudate and cerebellum (reference region), together with the kinetic modeling fit from dynamic two-parameter SRTM, for the $0.2 \mathrm{mg} / \mathrm{kg}$ quinpirole injections in animal M2. The simultaneously acquired CBV time course is shown in the bottom row. For the $0.1,0.2$, and $0.3 \mathrm{mg} / \mathrm{kg}$ quinpirole injections in animals $\mathrm{M} 1$ and (M2), the \%CBV signal change peaked at 3 (2.3), 2.3 (1.7), and $2.2(2.1) \mathrm{min}$, and returned to baseline within 15.1 (16.1), 8.5 (12.1), and 15.6 (14.5) min. Hence, for all doses, the \%CBV signal change peaked within a few minutes and returned to baseline quickly. The duration of the signal was defined as starting with the onset of the gamma variate GLM regressor until it returned to $<0.1 \%$ absolute CBV signal change. In addition to the short negative response, we observed that the $\mathrm{CBV}$ signal returned to a 

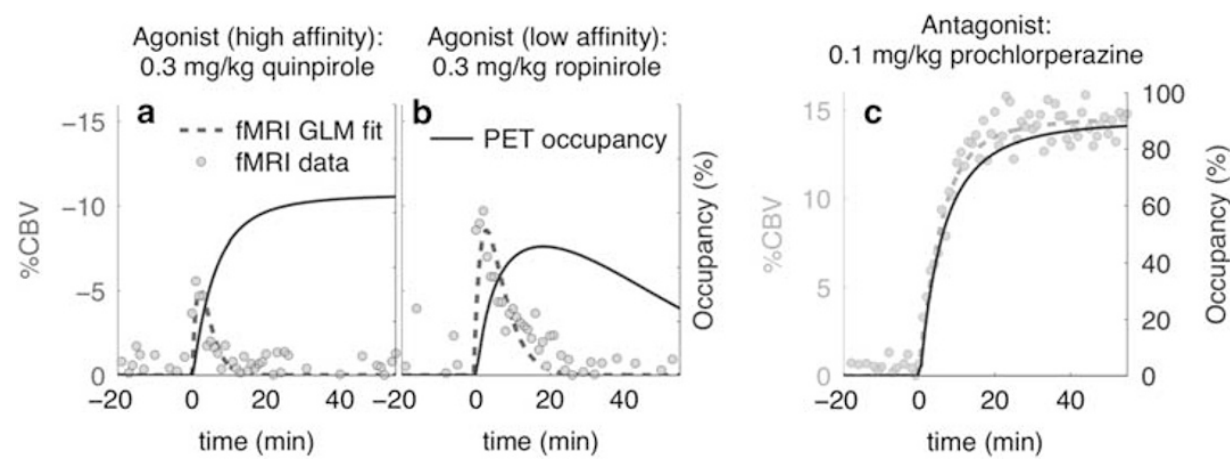

Figure 5 Time courses of CBV (dotted line) and occupancy (solid line) resulting from exposure to three different pharmacological challenges in animal M2: (a) the high-affinity agonist quinpirole elicits a very short CBV response, whereas PET occupancy stays elevated for the duration of the experiment. (b) The agonist ropinirole has a lower affinity compared with quinpirole and results in a slightly longer, though still short, CBV response, whereas occupancy peaks at $17.8 \mathrm{~min}$ and then starts to decrease. Compared with quinpirole, ropinirole displays a larger CBVPeak signal at lower occupancy. (c) The antagonist prochlorperazine shows that CBV and occupancy time courses are matched, and demonstrates that CBV can stay elevated for longer durations in time. The antagonist showed a reversed CBV sign and the largest magnitude compared with the agonists. Overall, the discrepancy in time between CBV and occupancy and diminished CBV magnitude for the agonists suggests that RDI affects both PET and fMRI, and can vary with drug affinity and potency. CBV, cerebral blood volume; fMRI, functional magnetic resonance imaging; GLM, general linear model; PET, positron emission tomography; RDI, receptor desensitization and internalization

positive value above its initial baseline. This slow rise in the CBV signal was modeled with a sigmoidal GLM regressor but was not included in the temporal profile as it was nonspecific to the striatum and observable in several brain regions. Contrary to the fast striatal CBV response, the PET signal decreased and remained low for the duration of the experiment in all cases. Kinetic modeling of the PET TACs with quinpirole challenges showed that changes in $\operatorname{DBP}(t)$ were better described by a sigmoidal function (according to the Akaike criterion) than any gamma variate function. Differences in Akaike values ranged from 0 to 29 (mean of 10) with the smallest AIC (best fit) being the sigmoidal fit in all cases. In cases where the difference in AIC was small, the gamma variate function was very close in shape to a sigmoidal curve.

Second quinpirole injections $(0.1$ and $0.2 \mathrm{mg} / \mathrm{kg}$ ) $70-120$ min after the first dose were administered to test whether the first response had been altered. In both experimental sessions with two doses, the second injection showed no detectable change in the fMRI signal (Figure 4), consistent with expectations of receptor internalization.

Ropinirole and prochlorperazine results. The temporal response from injections with lower-affinity agonist ropinirole $(0.1$ and $0.3 \mathrm{mg} / \mathrm{kg})$ showed robust negative CBV responses localized to caudate and putamen. Compared with quinpirole, the CBV time course was similar in shape but peaked slightly later, at 4.0 and $3.4 \mathrm{~min}$ after each dose injection (Figure $5 \mathrm{~b}$ ). The return to baseline was also slower, with the CBV response in caudate due to the $0.1 \mathrm{mg} / \mathrm{kg}$ ropinirole dose lasting $28.5 \mathrm{~min}$ and that due to the $0.3 \mathrm{mg} / \mathrm{kg}$ dose lasting $25.0 \mathrm{~min}$. The PET response for ropinirole showed that occupancy did not stay elevated but peaked at $17.8 \mathrm{~min}$ after which it decreased. In agreement with this, kinetic modeling showed that $\mathrm{DBP}(\mathrm{t})$ was better described with a gamma variate rather than sigmoidal function. The fMRI and PET time courses for ropinirole were thus less divergent than for quinpirole. In addition, the
$\mathrm{CBV}^{\text {peak }}$ magnitude from quinpirole was smaller, despite its higher potency in vitro, compared with ropinirole, consistent with predictions of the RDI model. Quinpirole showed a $\mathrm{CBV}^{\text {peak }}$ signal of $-5 \%$, whereas the less potent agonist ropinirole displayed a $\mathrm{CBV}^{\text {peak }}$ signal of $-8.5 \%$, despite reaching lower occupancy.

Prochlorperazine administration was employed as a control since, as a D2/D3 antagonist, we expect little or no RDI in this case. Figure $5 c$ shows that the CBV and occupancy measures match, suggesting that both time courses represent the dynamics of the injected drug itself at the postsynaptic membrane. Compared with the agonists, the CBV response from prochlorperazine resulted in the largest peak magnitude of $14.4 \%$ at $88 \%$ occupancy. These results are in concordance with the classical occupancy theory.

Estimation of desensitization and internalization constants. Fitting the proposed RDI model to experimental data, we estimated the in vivo RDI time constants $1 / \mathrm{k}_{\mathrm{DI}}$ to be $5 \pm 1$ min $($ mean $\pm S D)$ for the three doses of the D2/D3 agonist quinpirole. Estimates of $1 / \mathrm{k}_{\mathrm{DI}}$ for the lower-affinity D2/D3 agonist ropinirole are $8.5 \pm 2.1 \mathrm{~min}$.

\section{DISCUSSION}

We have shown that selective D2/D3 agonists elicited dose-dependent increases in receptor occupancy, together with decreases in CBV, in caudate and putamen of anesthetized NHPs. These spatial and dose correlations between changes in $\mathrm{D} 2 / \mathrm{D} 3$ receptor occupancy and changes in CBV support a neurovascular-coupling mechanism during receptor-specific activation. This relationship holds for D2/ D3 agonists, as shown in this study with quinpirole and ropinirole, as well as for D2/D3 antagonists, including prochlorperazine and raclopride (Sander et al, 2013b). Consistent with the known coupling of D2/D3 GPCR stimulation, we observed negative CBV changes in caudate and putamen, an in vivo measurement of receptor-specific 
functional inhibition. These results conform to positive CBV changes observed due to antagonism at $\mathrm{D} 2 / \mathrm{D} 3$, as previously reported (Sander et al, 2013b). Agonist-induced CBV changes observed outside of the striatum at high doses could indicate the activation of regions interconnected to the striatum or secondary effects of drug exposure.

Dynamic PET and fMRI signals after exposure to D2/D3 agonists (quinpirole, ropinirole) exhibited a pronounced temporal dissociation: although receptor binding of $\left[{ }^{11} \mathrm{C}\right]$ raclopride stayed decreased for a prolonged time, CBV signals returned to baseline rapidly, much faster than expected compared to the reported binding offset time of $\sim 20$ min for the agonist quinpirole (Levant et al, 1992). As PET and fMRI temporal responses were observed to be matched for D2/D3 antagonists and consistent with a classical occupancy model (Figure 5; Sander et al, 2013b), the agonist-induced temporal divergence suggests additional physiological mechanisms that modulate signals during agonist exposure.

Our proposed model suggests how RDI can produce measurable signal changes using concurrent PET/fMRI. Although the concept of RDI and evidence for it has been established in the literature, a quantitative model of RDI that integrates an in vivo functional readout with direct receptor measurements has been lacking. If receptors desensitize and internalize rapidly, our model predicts that PET and fMRI signals are driven in opposite directions from the expected binding and response profile of the drug (Figure 2). Our data are consistent with this RDI model, with the temporal divergence a measure of RDI rate that can be quantified. Both experimental data and our model suggest that higher $\mathrm{RDI}$ rates produce greater divergence.

\section{Evidence for In vivo RDI}

Several PET studies have suggested that a persistent decrease in PET signal is due to agonist-induced receptor internalization (Guo et al, 2009; Laruelle, 2000; Sun et al, 2003). Skinbjerg et al (2010) showed that an artificially prolonged increase in occupancy occurs in wild-type mice after amphetamine exposure but not in mice that have a knockout of the $\beta$-arrestin-2 gene (ARRB2), which is imperative for internalization. Suppression of the PET signal was still evident after $4 \mathrm{~h}$ in wild-type animals, whereas the signal had returned to baseline levels in ARRB2-knockout mice. This suggests that once receptors are internalized, this state may be preserved for several hours, with receptors being either recycled or degraded afterwards (Beaulieu and Gainetdinov, 2011). Our PET data using an exogenous agonist concurs with this interpretation as we observed a prolonged decrease in the availability of D2/D3 receptors.

The absence of an fMRI response with a second injection of the D2/D3 agonist quinpirole after $1-2 \mathrm{~h}$ is in agreement with electrophysiological recordings. These show that reapplication of quinpirole fails to initiate a second response in dopaminergic neurons of the ventral tegmental area (Bartlett et al, 2005). Although receptor signals can be transduced through the $\beta$-arrestin pathway (Lefkowitz and Shenoy, 2005), the functional consequences mainly include endocytosis and ERK activation, and we expect the magnitude and timing to be much less compared to the amplification of the G-protein-coupled pathway. We thus did not expect to observe a second response due to a second quinpirole injection. Moreover, D2Rs may undergo degradation post endocytosis (Bartlett et al, 2005), which can further contribute to the long recovery period of D2 receptor availability.

The D2/D3 agonist quinpirole has been shown to rapidly induce receptor internalization in vitro (Guo et al, 2009). As an initial step of this process, receptors desensitize due to phosphorylation, which decouples them from G-protein signaling and causes them to become functionally inactive. Because of this disconnect in the functional signaling chain, we expected fMRI signal to subside as receptors desensitize and that this effect would abbreviate the fMRI signal even when the drug was available for binding. All doses of quinpirole induced fMRI responses that reached peak magnitude within minutes and lasted $<30 \mathrm{~min}$, suggesting that RDI occurs rapidly in vivo.

Desensitization and internalization are closely linked mechanisms that allow dynamic regulation of cell signaling. Initial desensitization after agonist exposure occurs by phosphorylation of receptors through kinases (Seeman, 2013), which causes receptors to become functionally inactive. This desensitization mechanism would itself cause the fMRI signal to abbreviate, but could neither explain the prolonged binding decrease nor the lack of a second response after several hours as dephosporylation occurs on the same timescale of minutes (Williams et al, 2013). Rather, it has been shown that phosphorylation can be succeeded by recruitment of $\beta$-arrestin-2, triggering receptor internalization after initial desensitization (Beaulieu et al, 2011). Given the reported time frames of these mechanisms, fMRI may initially reflect desensitization, whereas PET may be more sensitive to long-term-binding properties, including internalization that lasts for hours, or even receptor degradation.

Alternative explanations to the RDI interpretation include the following. In in vitro settings, it has been shown that the D2R can exist in a high- and low-affinity state and can change between states in response to pharmacological injections (Seeman et al, 2006), which may explain our observations. The state of the receptor is determined by the affinity of DA to bind, but exogenous drugs seem to have identical affinity for high- and low-affinity receptors (Seeman, 2011). Although the existence of the two affinity states has been detected in homogenized tissues, in vivo states are less well established. Moreover, the high-affinity state is understood to be functionally active, whereas the lowaffinity state is linked to receptor internalization (Ko et al, 2002). The effects we observed on both PET and fMRI data may thus be the result of a change in affinity states, providing a way to image different affinity states of the $\mathrm{D} 2 / \mathrm{D} 3$ receptor.

The time course of the observed CBV signal on its own could be explained without RDI if quinpirole were a weak partial agonist, exhibiting a biphasic response within a classical occupancy model. However, this explanation cannot simultaneously account for the PET response, which should resolve towards baseline much more rapidly. Alternatively, the data could be explained by desensitization without internalization if quinpirole binds to synaptic D2/D3 receptors for the duration of the experiment. However, quinpirole binding to the receptor is not expected to last several hours, with $1 / k_{\text {off }}$ being $\sim 20$ min (Levant et al, 1992). In addition, there is considerable evidence from in vitro 
studies that quinpirole is an agonist causing receptor internalization with high functional potency (Gardner and Strange, 1998), and is used as a reference for evaluating the efficacy of other D2/D3 agonists (Coldwell et al, 1999; Perachon et al, 1999). The pieces of evidence presented in this study, together with current literature from in vitro data and in vivo imaging, strongly point towards receptor desensitization, followed by internalization, being the mechanism underlying our observations of divergence between apparent occupancy and function in vivo.

\section{Desensitization and Internalization Model for In vivo Quantification}

We investigated the effect of affinity changes of internalized receptors to available ligands in order to address the signal mechanisms underlying these non-invasive imaging modalities (Supplementary Information; Supplementary Figure 4). Although neurotransmitters such as DA do not passively cross cell membranes, PET ligands appear to access internalized receptors with altered affinity. Some data suggest that raclopride cannot access internalized receptors (Sun et al, 2003), whereas others report binding with a reduced affinity (Guo et al, 2009), which can lead to underestimation of occupancy (Seeman et al, 1989). If the affinity of raclopride for internalized receptors does not change, then the PET signal is a measure of total receptor availability, although a subset of these receptors would be functionally inactive. But if the affinity to internalized receptors is reduced, then a component of observed elevated occupancy reflects a low affinity rather than increased binding density. Conversely, if internalization increases the affinity for the radioligand by preventing efflux more than influx of the radioligand, then PET could report druginduced reductions in apparent receptor occupancy (Supplementary Figure 4). This scenario has been hypothesized as an explanation for paradoxical results obtained using spiperone (Chugani et al, 1988). Differences between spiperone binding and raclopride binding could be interpreted either as spiperone but not raclopride being able to access internalized receptors or spiperone having increased affinity to internalized receptors and raclopride-reduced affinity (Trevor Young et al, 1991). In either case, raclopride would have reduced affinity to internalized receptors, consistent with our observations.

The RDI model predicts that CBV peak magnitudes decrease with higher RDI rates, consistent with our experimental data. Although quinpirole has higher affinity than ropinirole, it produced a smaller $\mathrm{CBV}$ magnitude at higher occupancy. Together with a later CBV peak and slower return to baseline for ropinirole, this suggests that RDI is less pronounced compared with the higher-affinity agonist quinpirole. This is supported by our rate estimates, according to which quinpirole has a shorter RDI time constant. The RDI rate-dependent magnitude changes can thus modify the CBV versus occupancy relationship, which may provide an additional indication (apart from temporal dissociations) for differentiating RDI rates among agonists.

Dynamic simultaneous PET/fMRI measurements combined with the appropriate multimodal signal model enable the measurement of RDI in vivo and a quantitative estimate of rates, which to date have not been measured in vivo to our knowledge. Comparing our model with the experimental data, in vivo RDI rates of quinpirole are $\sim 0.2 / \mathrm{min}$. This is consistent with in vitro measurements of internalization time constants, which are reported to be on the order of $5 \mathrm{~min}$ (Guo et al, 2009). Although these results suggest that in vivo and in vitro RDI rates are on the same order of magnitude, other conditions, such as the recycling of receptors, may affect and alter RDI rates in vivo. However, in the absence of any other in vivo measurements, our comparisons are limited to in vitro rates. Our study provides a starting point to investigate the timescales of these mechanisms in an in vivo setting.

\section{Study Limitations and Future Extensions}

The magnitude of binding potentials before and after drug challenges could be affected by animals being anesthetized. It has been shown previously that pharmacological challenges administered under $1.5-2 \%$ isoflurane anesthesia result in a larger decrease in binding potential when compared with awake animals (Tsukada et al, 1999). Continuous infusion of ketamine itself can decrease striatal binding of $\left[{ }^{11} \mathrm{C}\right]$ raclopride and thus affect PET quantification (Tsukada et al, 2000). In our study, we applied a single dose of ketamine injection and kept isoflurane to $\sim 1 \%$, so that we expect these effects to be small. Although absolute magnitudes may be affected, we do not expect our study outcomes to change due to anesthesia as they largely depend on withinanimal comparisons.

Our imaging data cannot provide direct evidence for RDI mechanisms. But considering the pieces of evidence and their discussion outlined above, the current literature, and the absence of any other known mechanisms that could explain the discrepancy we observed in our data, RDI is likely to have a strong role. In light of neurovascular coupling to occupancy occurring for antagonists, we suggest that desensitization and internalization are the most parsimonious mechanisms that can explain both the CBV and occupancy data, and fit with all known characteristics associated with RDI kinetics and molecular biology. Future extension of this work can be directed towards investigating and comparing other pharmacological compounds that are well-characterized in vitro, to serve as a basis for interpreting in vivo dynamics. Moreover, imaging genetically modified animals that cannot downregulate D2/D3 receptors (eg, ARRB2 knockouts as in Skinbjerg et al, 2010) and comparing their CBV-occupancy time courses due to agonist injection to wild-type animals would provide more direct evidence of RDI mechanisms. Studies like that could even delineate a component of the CBV response that correlates with receptor downregulation.

\section{Neuroscientific and Clinical Implications}

Measuring RDI dynamics in vivo is important in order to elucidate drug responses and to establish links to behavior. Repeated exposure to agonists in rodents and NHPs has been linked to supersensitivity (Bolaños-Jiménez et al, 2011) and this phenomenon has been suggested to have a role in the development of schizophrenia (Seeman and Seeman, 2014). However, to date, it is challenging to directly compare acute effects of high-dose agonist exposure directly with existing 
rodent behavioral studies, which typically differ by the dose and route of administration. Behavioral patterns change versus time and dose, with biphasic patterns attributed to pre- $v s$ postsynaptic function (Arnsten $e t$ al, 1995; Eilam and Szechtman, 1989) or to a balance between excitatory and inhibitory stimulation (Winstanley et al, 2010). Carrying out imaging studies that combine readouts of occupancy, function, and behavior in awake animals could shed light on these patterns and their link to RDI.

Receptor states and dynamics may be affected in disease and further modified through drug therapy. Full or partial D2 agonists are approved for the treatment of, eg, movement disorders and psychiatric disease but it is unknown how both short- and long-term exposure of drugs affects receptor dynamic function. The dynamic function of other receptor systems has also been suggested to be affected by RDI. The $5-\mathrm{HT}_{2 \mathrm{~A}}$ receptor system has shown similar observations as the D2/D3 receptor system that can be attributed to internalization, though further insight into RDI would be needed (Gründer et al, 1997). The regulation of receptor trafficking in, eg, the glutamate system has been linked to schizophrenia (Hall et al, 2015), and altered serotonin and DA levels have been suggested to affect $5-\mathrm{HT}_{2 \mathrm{~A}}$ internalization (Bhattacharyya et al, 2006), thus having an important role for drug treatments in anxiety and depression. The ability to measure RDI dynamics in vivo could help clarify disease mechanisms, advance therapeutic treatment, and evaluate drug efficacy in neurological and neuropsychiatric disorders.

\section{FUNDING AND DISCLOSURE}

This research was supported by grants from the National Institutes of Health: R90DA023427, P41EB015896, S10RR026666, S10RR022976, S10RR019933 and S10RR017 208. The authors declare no conflict of interest.

\section{ACKNOWLEDGMENTS}

We thank Grae Arabasz, Shirley Hsu, Helen Deng, Avilash Cramer, Judit Sore, Kari Phan, and Garima Gantam for their help and work on radiotracer synthesis, coil design, animal preparation, and PET/MR scanning.

\section{REFERENCES}

Alpert NM, Badgaiyan RD, Livni E, Fischman AJ (2003). A novel method for noninvasive detection of neuromodulatory changes in specific neurotransmitter systems. NeuroImage 19: 1049-1060.

Arnsten AF, Cai JX, Steere JC, Goldman-Rakic PS (1995). Dopamine D2 receptor mechanisms contribute to age-related cognitive decline: the effects of quinpirole on memory and motor performance in monkeys. J Neurosci 15: 3429-3439.

Bartlett SE, Enquist J, Hopf FW, Lee JH, Gladher F, Kharazia V et al (2005). Dopamine responsiveness is regulated by targeted sorting of D2 receptors. Proc Natl Acad Sci USA 102: 11521-11526.

Beaulieu J-M, Del' Guidice T, Sotnikova T, Lemasson M, Gainetdinov R (2011). Beyond cAMP: the regulation of Akt and GSK3 by dopamine receptors. Front Mol Neurosci 4: 38.

Beaulieu J-M, Gainetdinov RR (2011). The physiology, signaling, and pharmacology of dopamine receptors. Pharmacol Rev 63: 182-217.
Bhattacharyya S, Raote I, Bhattacharya A, Miledi R, Panicker MM (2006). Activation, internalization, and recycling of the serotonin 2A receptor by dopamine. Proc Natl Acad Sci USA 103: $15248-15253$.

Bolaños-Jiménez R, Arizmendi-Vargas J, Martínez-Zavala N, Carrillo-Ruiz J, Damián CÁ-TJ, Luis J-PF et al (2011). Quinpirole effects on the dopaminergic system. Br J Pharmacol Toxicol 2: 310.

Breier A, Su T-P, Saunders R, Carson RE, Kolachana BS, de Bartolomeis A et al (1997). Schizophrenia is associated with elevated amphetamine-induced synaptic dopamine concentrations: Evidence from a novel positron emission tomography method. Proc Natl Acad Sci USA 94: 2569-2574.

Carson RE, Channing MA, Blasberg RG, Dunn BB, Cohen RM, Rice KC et al (1993). Comparison of bolus and infusion methods for receptor quantitation: application to [18f]cyclofoxy and positron emission tomography. J Cereb Blood Flow Metab 13: 24-42.

Catana C, van der Kouwe A, Benner T, Michel CJ, Hamm M, Fenchel $\mathrm{M}$ et al (2010). Toward implementing an MRI-based PET attenuation-correction method for neurologic studies on the MRPET brain prototype. J Nucl Med 51: 1431-1438.

Chen YCI, Galpern WR, Brownell A-L, Matthews RT, Bogdanov M, Isacson O et al (1997). Detection of dopaminergic neurotransmitter activity using pharmacologic MRI: correlation with PET, microdialysis, and behavioral data. Magn Reson Med 38: 389-398.

Chen YI, Brownell AL, Galpern W, Isacson O, Bogdanov M, Beal MF et al (1999). Detection of dopaminergic cell loss and neural transplantation using pharmacological MRI, PET and behavioral assessment. Neuroreport 10: 2881-2886.

Choi J-K, Chen YI, Hamel E, Jenkins BG (2006). Brain hemodynamic changes mediated by dopamine receptors: role of the cerebral microvasculature in dopamine-mediated neurovascular coupling. NeuroImage 30: 700-712.

Chugani DC, Ackermann RF, Phelps ME (1988). In vivo [3H] spiperone binding: evidence for accumulation in corpus striatum by agonist-mediated receptor internalization. J Cereb Blood Flow Metab 8: 291-303.

Clark AJ (1937). General Pharmacology. Springer-Verlag: Berlin, New York.

Coldwell MC, Boyfield I, Brown T, Hagan JJ, Middlemiss DN (1999). Comparison of the functional potencies of ropinirole and other dopamine receptor agonists at human $\mathrm{D}_{2 \text { (long) }}, \mathrm{D}_{3}$ and $\mathrm{D}_{4.4}$ receptors expressed in Chinese hamster ovary cells. $\mathrm{Br} J$ Pharmacol 127: 1696-1702.

Cox RW (2012). AFNI: what a long strange trip it's been. Neuroimage 62: 743-747.

Cumming P (2011). Absolute abundances and affinity states of dopamine receptors in mammalian brain: a review. Synapse 65: 892-909.

Eilam D, Szechtman H (1989). Biphasic effect of D-2 agonist quinpirole on locomotion and movements. Eur J Pharmacol 161: 151-157.

Gardner B, Strange PG (1998). Agonist action at D2(long) dopamine receptors: ligand binding and functional assays. $\mathrm{Br} J$ Pharmacol 124: 978-984.

Ginovart N (2005). Imaging the dopamine system with in vivo [11C]raclopride displacement studies: understanding the true mechanism. Mol Imaging Biol 7: 45-52.

Goggi JL, Sardini A, Egerton A, Strange PG, Grasby PM (2007). Agonist-dependent internalization of D2 receptors: imaging quantification by confocal microscopy. Synapse 61: 231-241.

Griswold MA, Jakob PM, Heidemann RM, Nittka M, Jellus V, Wang J et al (2002). Generalized autocalibrating partially parallel acquisitions (GRAPPA). Magn Reson Med 47: 1202-1210.

Gründer G, Yokoi F, Offord SJ, Ravert HT, Dannals RF, Salzmann JK et al (1997). Time course of 5-HT2A Receptor occupancy in the human brain after a single oral dose of the 
putative antipsychotic drug MDL 100,907 measured by positron emission tomography. Neuropsychopharmacology 17: 175-185.

Guo N, Guo W, Kralikova M, Jiang M, Schieren I, Narendran R et al (2009). Impact of D2 receptor internalization on binding affinity of neuroimaging radiotracers. Neuropsychopharmacology 35: 806-817.

Hall J, Trent S, Thomas KL, O’Donovan MC, Owen MJ (2015). Genetic risk for schizophrenia: convergence on synaptic pathways involved in plasticity. Biol Psychiatry 77: 52-58.

Ichise M, Liow J-S, Lu J-Q, Takano A, Model K, Toyama $\mathrm{H}$ et al (2003). Linearized reference tissue parametric imaging methods: application to [11C]DASB positron emission tomography studies of the serotonin transporter in human brain. J Cereb Blood Flow Metab 23: 1096-1112.

Ko F, Seeman P, Sun WS, Kapur S (2002). Dopamine D2 receptors internalize in their low-affinity state. Neuroreport 13: 1017-1020.

Lammertsma AA, Hume SP (1996). Simplified reference tissue model for PET receptor studies. Neuroimage 4: 153-158.

Lane DA, Chan J, Fitzgerald ML, Kearn CS, Mackie K, Pickel VM (2012). Quinpirole elicits differential in vivo changes in the preand postsynaptic distributions of dopamine D2 receptors in mouse striatum: relation to cannabinoid-1 (CB1) receptor targeting. Psychopharmacology (Berl) 221: 101-113.

Laruelle M (2000). Imaging synaptic neurotransmission with in vivo binding competition techniques: a critical review. J Cereb Blood Flow Metab 20: 423-451.

Laruelle M, Iyer RN, Al-Tikriti MS, Zea-Ponce Y, Malison R, Zoghbi SS et al (1997). Microdialysis and SPECT measurements of amphetamine-induced dopamine release in nonhuman primates. Synapse 25: 1-14.

Lefkowitz RJ, Shenoy SK (2005). Transduction of receptor signals by ß-arrestins. Science 308: 512-517.

Levant B, Grigoriadis DE, DeSouza EB (1992). Characterization of $[3 \mathrm{H}]$ quinpirole binding to D2-like dopamine receptors in rat brain. J Pharmacol Exp Ther 262: 929-935.

Maehle A-H, Prüll C-R, Halliwell RF (2002). The emergence of the drug receptor theory. Nat Rev Drug Discov 1: 637-641.

Mandeville JB (2012). IRON fMRI measurements of CBV and implications for BOLD signal. Neuroimage 62: 1000-1008.

Mandeville JB, Marota JJA, Kosofsky BE, Keltner JR, Weissleder R, Rosen BR et al (1998). Dynamic functional imaging of relative cerebral blood volume during rat forepaw stimulation. Magn Reson Med 39: 615-624.

Mandeville JB, Sander CYM, Jenkins BG, Hooker JM, Catana C, Vanduffel W et al (2013). A receptor-based model for dopamineinduced fMRI signal. Neuroimage 75: 46-57.

McLaren DG, Kosmatka KJ, Oakes TR, Kroenke CD, Kohama SG, Matochik JA et al (2009). A population-average MRI-based atlas collection of the rhesus macaque. Neuroimage 45: 52-59.

Narendran R, Slifstein M, Guillin O, Hwang Y, Hwang D-R, Scher E et al (2006). Dopamine (D2/3) receptor agonist positron emission tomography radiotracer [11C]-(+)-PHNO is a D3 receptor preferring agonist in vivo. Synapse 60: 485-495.

Normandin MD, Schiffer WK, Morris ED (2012). A linear model for estimation of neurotransmitter response profiles from dynamic PET data. Neuroimage 59: 2689-2699.

Perachon S, Schwartz J-C, Sokoloff P (1999). Functional potencies of new antiparkinsonian drugs at recombinant human dopamine D1, D2 and D3 receptors. Eur J Pharmacol 366: 293-300.

Saleem KS, Logothetis NK (2006). A Combined MRI and Histology Atlas of the Rhesus Monkey Brain. Elsevier: Burlington, USA.

Sander CY, Cramer A, Keil B, Mareyam A, Rosen BR, Wald LL (2013a). Low $511 \mathrm{keV}$-attenuation array coil setup for simultaneous PET/MR imaging of the monkey brain. Proc Intl Soc Mag Reson Med 21.

Sander CY, Hooker JM, Catana C, Normandin MD, Alpert NM, Knudsen GM et al (2013b). Neurovascular coupling to D2/D3 dopamine receptor occupancy using simultaneous PET/functional MRI. Proc Natl Acad Sci USA 201220512.

Seeman MV, Seeman P (2014). Is schizophrenia a dopamine supersensitivity psychotic reaction? Prog Neuropsychopharmacol Biol Psychiatry 48: 155-160.

Seeman P (2011). All roads to schizophrenia lead to dopamine supersensitivity and elevated dopamine D2high receptors. CNS Neurosci Ther 17: 118-132.

Seeman P (2013). Are dopamine D2 receptors out of control in psychosis? Prog Neuropsychopharmacol Biol Psychiatry 46: 146-152.

Seeman P, Guan H-C, Niznik HB (1989). Endogenous dopamine lowers the dopamine D2 receptor density as measured by [3H] raclopride: implications for positron emission tomography of the human brain. Synapse 3: 96-97.

Seeman P, Wilson A, Gmeiner P, Kapur S (2006). Dopamine D2 and D3 receptors in human putamen, caudate nucleus, and globus pallidus. Synapse 60: 205-211.

Skinbjerg M, Liow J-S, Seneca N, Hong J, Lu S, Thorsell A et al (2010). D2 dopamine receptor internalization prolongs the decrease of radioligand binding after amphetamine: a PET study in a receptor internalization-deficient mouse model. Neuroimage 50: $1402-1407$.

Sokoloff P, Giros B, Martres M-P, Bouthenet M-L, Schwartz J-C (1990). Molecular cloning and characterization of a novel dopamine receptor (D3) as a target for neuroleptics. Nature 347: $146-151$.

Sun W, Ginovart N, Ko F, Seeman P, Kapur S (2003). In vivo evidence for dopamine-mediated internalization of D2-receptors after amphetamine: differential findings with $[3 \mathrm{H}]$ raclopride versus [3H]spiperone. Mol Pharmacol 63: 456-462.

Trevor Young L, Wong DF, Goldman S, Minkin E, Chen C, Matsumura $\mathrm{K}$ et al (1991). Effects of endogenous dopamine on kinetics of $[3 \mathrm{H}] \mathrm{N}$-methylspiperone and $[3 \mathrm{H}]$ raclopride binding in the rat brain. Synapse 9: 188-194.

Tsukada H, Harada N, Nishiyama S, Ohba H, Sato K, Fukumoto D et al (2000). Ketamine decreased striatal [11C]raclopride binding with no alterations in static dopamine concentrations in the striatal extracellular fluid in the monkey brain: multiparametric PET studies combined with microdialysis analysis. Synapse 37: 95-103.

Tsukada H, Nishiyama S, Kakiuchi T, Ohba H, Sato K, Harada N (1999). Is synaptic dopamine concentration the exclusive factor which alters the in vivo binding of [11C]raclopride?: PET studies combined with microdialysis in conscious monkeys. Brain Res 841: $160-169$.

Williams JT, Ingram SL, Henderson G, Chavkin C, von Zastrow M, Schulz S et al (2013). Regulation of $\mu$-opioid receptors: desensitization, phosphorylation, internalization, and tolerance. Pharmacol Rev 65: 223-254.

Winstanley CA, Zeeb FD, Bedard A, Fu K, Lai B, Steele C et al (2010). Dopaminergic modulation of the orbitofrontal cortex affects attention, motivation and impulsive responding in rats performing the five-choice serial reaction time task. Behav Brain Res 210: 263-272.

Worsley KJ, Liao CH, Aston J, Petre V, Duncan GH, Morales F et al (2002). A general statistical analysis for fMRI data. Neuroimage 15: $1-15$.

Supplementary Information accompanies the paper on the Neuropsychopharmacology website (http://www.nature.com/npp) 